\title{
Artificial Intelligency, Big Data and Cultural Heritage
}

\author{
Giorgio Buratti \\ Sara Conte \\ Michela Rossi
}

Abstract

In recent decades, the cultural heritage sector has benefited from solutions offered by ICT for the conservation, management, enhancement and communication of cultural heritage; today this specific sector benefits from the infinite potential of application of Al. The proposed research identifies the three main lines of research that operate in the cultural heritage exploiting the synergy between machine learning, big data and Al, starting from the analysis of the state of the art and a subsequent first taxonomic approximation of artificial intelligence systems. The analysis of some case studies developed in the field of recovery and restoration of cultural heritage, monitoring and prevention of damage, data acquisition and analysis of the same, confirm the real potential of Al: trigger knowledge from knowledge.

Keywords

Al, cultural heritage, interaction, automatic drawing, taxonomy.

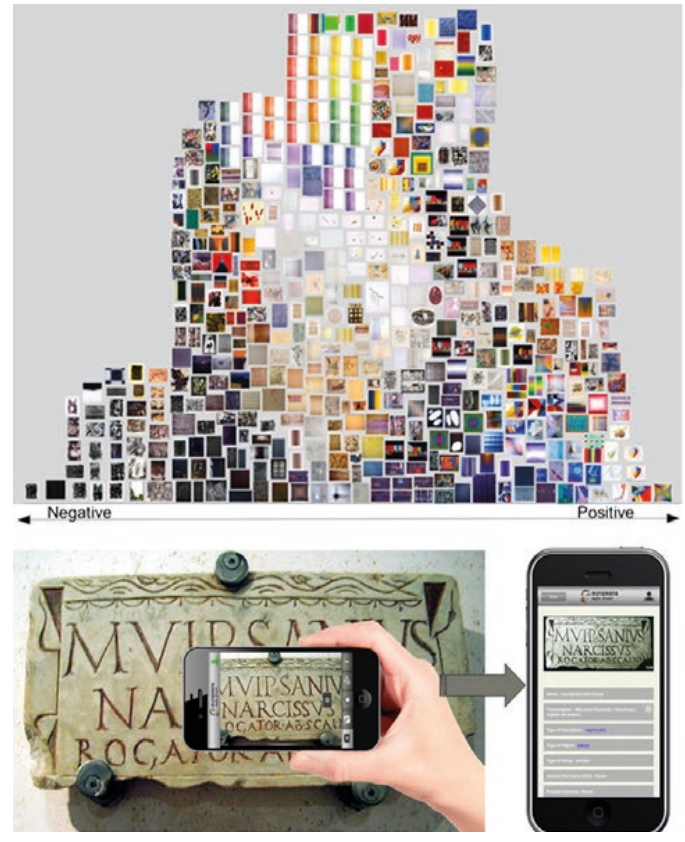

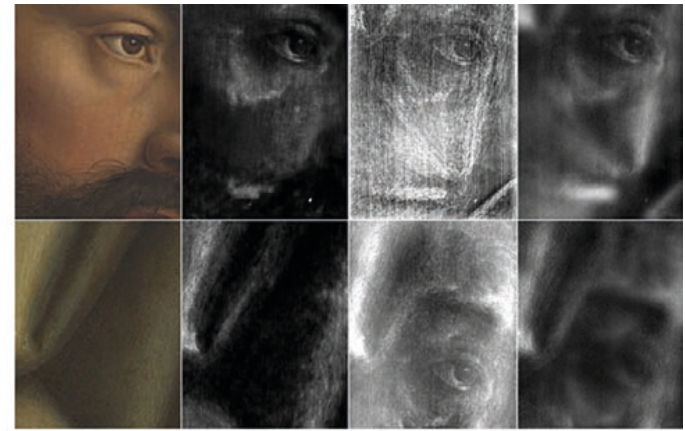

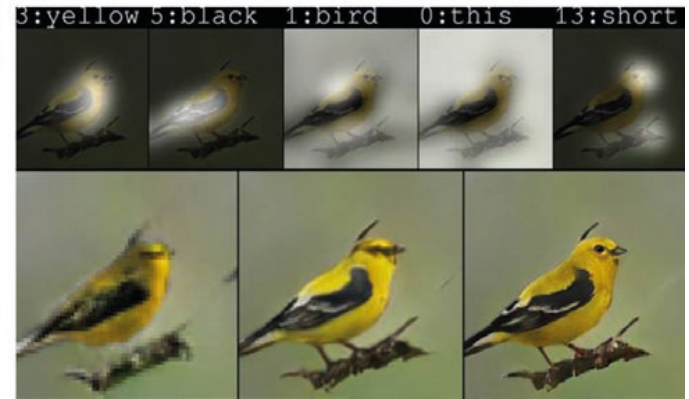




\section{Introduction}

Since the 2000s, the improvement in microchips' computing performance has made creating, collecting and transmitting data so fast and cheap, to bring the amount of available information to exceed a crucial threshold [I], determining the advent of the so-called Big Data era. Originally, the term "Big Data" referred to the technical ability to collect, store and process increasing amounts of data at decreasing costs. There is nothing innovative about this definition, which could be compared to the advantages of printing over handwriting or any other technical improvement in cultural technologies history. The unprecedented aspect is the ease of accessing more data than it seems possible to handle for the first time in human history data is abundant and cheap and continues to exponentially increase.The information collected in digital form in a delocalized telecommunications network is accessible in real time to various types of electronic devices that allow data re-processing. In this context, any search creates in a few seconds a redundant mass of information that complicates the identification of the most significant content. The quantity and type of information has reached a complexity that transcends a direct management possibility, requiring an adaptation of the taxonomic ordering structures used up to now to understand phenomenal reality and to manage knowledge.

Fig. I. Taxonomy of Ai referred to F. Corea, https://www.kdnuggets. com

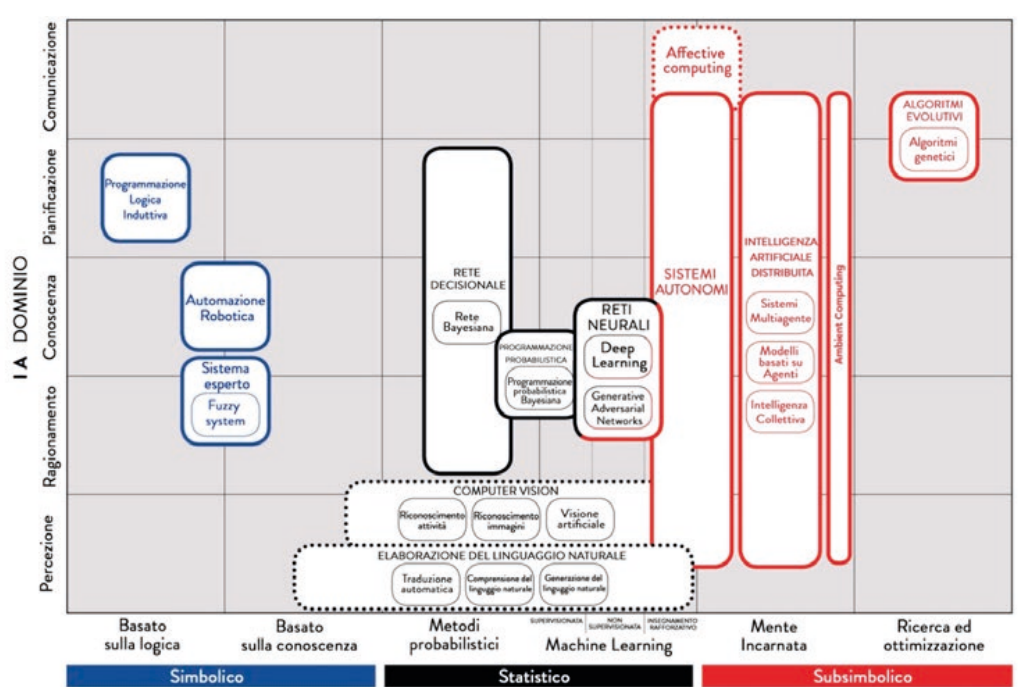

APPLICAZIONI SPECIFICHE
O APPLICAZIONI GENERALI sotTotipI

IA TASSONOMIA

\section{Al and Big Data: Order Complexity}

Unlike humans, computers can use the growing amount of 'disordered' information by defining a new way of producing knowledge, no longer based on reference theories, but on a posteriori identification of recurring patterns within the rough data flow. This abdication of the causal logic linked to reasoning in favor of computational analysis does not imply a 'technological' determinism or that data driven research must guide all cognitive processes, because the data has no intrinsic value but in a defined context. If knowledge's inferential processes start from the informations acquired, it's also true that a simple data accumulation does not generate knowledge without a method capable of identifying the correlations among variables. In this context becomes crucial research on Artificial Intelligence, a definition that includes a differentiated set of techniques and methods with the aim of automating human cognitive abilities. And since computers process data at speeds now close to the speed of light [2], it is possible to analyze increasing amounts of information, generating predictive models based on objective assessments. Since its inception, Al research focuses on the creation of models that simulate the dynamics of human intelligence, based on the comparison and reworking of external stimuli, which can be classified according to the main active research directions. The table (fig. I) shows on ordinate axis the abilities of human intelligence: 
- Perception: skill to transform sensory inputs into useful information);

- Reasoning: skill to connect / solve problems;

- Communication: ability to elaborate a language and transmit concepts;

- Knowledge: skill to understand and represent reality;

- Planning:skill to define and achieve objectives.

The main research lines currently recognized are summarized on the abscissa axis:

- Symbolic Approach: which considers intelligence as that process of transforming perceptual stimuli into symbols to which a meaning can be attributed, according to more or less formal rules;

- Statistical Approach: based on logical-mathematical tools to extract information content useful for transforming information into knowledge starting from Bayesian inference, formalizing the hypothesis that a greater use of a given data increases the probability of its exactness; - Sub-Symbolic Approach: based on neuronal functioning whereby information is transmitted regardless of meanings: knowledge emerges regardless of previous mental models, but on the basis of structures based on experience.

From these line of research, six subgroups descend which identify the main Al applications: - Logic-based: tools used for problem solving through the orderly representation of knowledge; - Knowledge-based: tools with extended ontologies and relational databases of notions, information and rules;

- Probabilistic Methods: tools that allow agents to act even in incomplete information scenarios;

- Machine Learning: tools that generate data from other data;

- Embodied Intelligence: engineering toolbox, which assumes that a body (or at least a partial set of functions such as movement, perception, interaction, and visualization) is required for higher intelligence

- Research and Optimization: predictive calculation tools that allow you to optimize possible solutions according to the specific context.

These methods are already used, often unknowingly, on a daily basis: when we ask for directions to our portable device or ask a virtual assistant to turn on the light, we are interacting with natural language interpretation Al and neural networks trained to carry out research and home automation operations.

For some years now, the use of genetic or evolutionary algorithms in form finding studies has consolidated the Al tools also in the drawing discipline. These processes consider the different morphological hypotheses as biological individuals and recombine them, similarly to sexual biological reproduction, introducing elements of disorder that simulate random genetic mutations. Thousands of formal solutions (new individuals) are thus generated, among which those that guarantee the highest level of optimization are chosen, just as selective processes promote the survival of the individuals most suitable for an ecosystem. In this way it is possible to explore solutions hitherto unused due to the lack of description and control tools adequate to the problem complexity.

\section{The New Frontiers of Al Applied to Cultural Heritage}

The first one collects all the research characterized by the development of innovative methodologies and systems to monitor and prevent damage to the cultural heritage, helping to increase the offer for the users. The most common example is the biosensor that can prevent damage from biological pests. This is any analog or digital device that works with living organisms (indicators) able to respond to certain environmental stimuli, producing an electro-chemical, optical signal to reach a threshold value.Today automatic biosensors are studied, that work in real time and continuously to signal in indoor environments the achievement of favorable conditions to germinative factors and therefore a constant monitoring of the state of health of the good [Danese et al. 2019]. Significant are the researches based on the use of neural networks in conservative perspective; in particular the one carry on by the researchers of National Gallery, Duke University and University College of London, that used neural networks to analyze complex high-resolution digital X-ray images of the cathedral of Gand "Adoration of the Mystical Lamb" [Van der Snickt et al. 2020]. Convolutional neural networks (CNN) combined with multi-resolution imaging techniques, initially used to determine the 
authorship of a work and to distinguish fakes, are now able to reconstruct unfinished or deteriorated drawings from time as demonstrated by the research of the Dutch Technical University of Delft [Zeng et al. 2019]. The goal is the automatic reconstruction, through a pixel wise technology, of Vincent van Gogh's drawings that are deteriorated by time. The team was able to return works now irretrievably lost to the public, using the algorithm inspired by biological neural networks similar to human brain ones and trained on a data set containing reproductions of original drawings of various qualities, sizes, made at different times.

The second area analyses the experiments that focus on automatic data acquisition, such as the application of mobile technologies for three-dimensional surveying and the acquisition of images of monuments, architectures, archaeological sites. The distributed Al is an interesting line of research that is giving the first results in the field of three-dimensional and photogrammetric relief, through drones that are able to direct their flight in strategies similar to the ones of bees and other social insects. Flight trajectories are not pre-programmed for individual units, but depend on the network of interactions given by the continuous communication between the various subjects, and between them and the environment in which they operate, as it is visible in the Aerial Construction project [Willmann et al. 2008]. The project of the DISI of the University of Trento in collaboration with local authorities, such as the MART of Rovereto goes further, developing $\mathrm{Al}$ able to analyze the influence on perceptive properties and the emotional level that a work of art arouses in the observer. The Al recognizes the intensity and the type of the emotion through a facial recognition system based on the affective computing and the deep learning, providing useful information for the museum and curators [Sartori et al. 20I6]. The work of the CNR-IMATI within the project Horizone 2020, GRAVITATE provides us an example of automatic extraction of features through the Generative adversarial network. The experimentation allows to identify the ceramic fragments, through the combination of semantic and geometric elements, and to assemble them in a semi-automatic way, simplifying the work of the archaeologist and giving the possibility to virtually reunify a spread archaeological heritage. [Moscoso Thompson, Biasotti 2019].

The third field aims to develop systems for the management of large amounts of data and informations, analog or digital, contained in libraries, archives, museums, and to provide the user with easier and more immediate access to information. There are many apps today that want to meet the needs of the users, who are visiting cities and monuments, by providing in real time useful and richer content and interpretations of our past, mainly using the ambient computing and the deep learning. Time Machine is a European project that aims at 4D digital reconstruction of every historical place of the main European cities, combining them with data of all types accessible with augmented reality interfaces; Monugram allows to give information of a monument based on the recognition of the image taken by the user in real time; Woolysses is an interactive chatbot for tours enriched with personalized multimedia content and anticipating the needs of the user; Eagle, allows automatic recognition of Greek/Latin epigraphs and dissemination of associated information. The aim of the different methodologies is unambiguous: to orient the technology towards the rediscovery of the knowledge to protect it or make it accessible, putting the digital reality at the service of the material one.

\section{Conclusion}

The fields of application of Al are constantly evolving and searching for new contexts in which the application of predictive algorithms allows the extrapolation of finalized solutions from the automatic reprocessing of a large amount of data. The intelligence of the machine is a consequence of the research speed of comparable information in a network that mimics neural connections. The solution is the statistical result of a comparison based on the evidence: a digital reinvention of the empiricism that preceded the development of experimental science, which built knowledge on reasoning aided by intuition. New knowledge is born from the empirical reworking of previous knowledge; the added value is parametric definition of reworking and the relationships between algorithms, controlled by humans. The inventiveness that distinguishes it does not depend only on logical capabilities; in fact the 
described context opens to a series of reflections within the transformations taking place in drawing and in the contribution of the machine to design.

For example, the Drawing-Bot is an Al developed by Microsoft in collaboration with three American universities that uses the technology of the antagonistic generative network to create images from its description. A neural generative network creates new images from some keywords, while an antagonistic network compares them with a database of existing images, eliminating the results that differ from it. At present this Al produces good results for short and explanatory expressions; it is able to easily extract the forms and then integrates them autonomously with the absent details from the given description, while the final result is likely to be a confused image for complex descriptions, but it is only a matter of time and speed of calculation. In the example [Zhang et al. 2017] the quality of the representation is close to the photographic one, but the reproduced bird does not exist in the reality, is a representation born from the "imagination" of a computer. This case opens up to a series of reflections on the machine's learning potential and on how the relationship between the machine and the designer may evolve in the future. Today the machine is able to create a drawing based on the same geometric rules used to trace it by hand, but will it be possible to teach the machine the capacity of autonomous reasoning and to simulate the human creative capacity, through data processing?

\section{Notes}

[I] At the time of writing [202I] the amount of data currently engaged in the world each year is about 40 ZB [zettabytes] One ZB corresponds to 102121 bytes, equivalent to approximately 180 million times the information collected in the records kept in the Library of Congress in Washington, recognized as the largest existing library with over 158 million documents held.

[2] At the time of writing, the most powerful computer in the world is Summit, developed by IBM for the ORNL, United States Department of Energy, capable of handling 100 petaflops, equivalent to 100 million billion operations per second. Summit is used in studies ranging from particle physics to weather/climate forecasts, from the creation of new drugs to the simulation of the effects of nuclear explosions.

[3] Although the paper was conceived jointly, Michela Rossi is the author of Introduction, Gorgio Buratti is the author of Al and Big Data: Order complexity and the related image, Sara Conte is the author of The new frontiers of Al applied to cultural heritage. The Conclusions are drafted jointly.

\section{References}

Andreza Sartori, Dubravko Culibrk, Yan Yan, Remo Job, e Nicu Sebe (20 I6). Computational modeling of affective qualities of abstract paintings. In IEEE MultiMedia, 23 (3), pp. 44-54.

Danese Maria, Sileo Maria, Rosa Lasaponara, Nicola Masini (2019). Proximal remote sensing e spatial analysis per la conservazione delle pitture parietali pompeiane. II caso del gymnasium. In ArcheomaticA, 2, pp. I2- 18.

Moscoso Thompson Elia, Biasotti Silvia (2019). Retrieving color patterns on surface meshes using edgelbp descriptors. In Computers \& Graphics, 79, pp. 46-57.

Van der Snickt Geert, Dooley Kathryn, Jana Sanyova, Hélène Dubois, Delaney John, Gifford Melanie, Legrand Stijn, Laquiere Nathalie, Janssens Koen (2020). Dual mode standoff imaging spectroscopy documents the painting process of the Lamb of God in the Ghent Altarpiece by J. and H.Van Eyck. In Science Advances, AAAS, 6 (31), pp. I - 0.

Willmann Jan, Augugliaro Federico, CadalbertThomas, D'Andrea Raffaello, Gramazio Fabio, Kohler Matthias (2008). Aerial Robotic. Construction towards a new field of architectural research. In International journal of architectural computing, 03 ( 10), pp. 439-459.

Zhang Han, Xu Tao, Li Hongsheng, Zhang Pengchuan, Wang Xiaogang, Huang Xaolei, Metaxas Dimitris (20 I7). Stackgan: Text to photo-realistic image synthesis with stacked generative adversarial networks. IEEE Int. Conf. on Computer Vision, pp. 907-59।

Zeng Yuan, van der Lubbe Jan, Loog Marco (2019). Multi-scale convolutional neural network for pixel-wise reconstruction of Van Gogh's drawings. In Machine Vision and Applications, Cham: Springer, pp. I 229- 124 I.

\section{Authors}

Giorgio Buratti, Dept. of Design, Politecnico di Milano, giorgio.buratti@polimi.it

Sara Conte, Dept. of Design, Politecnico di Milano, sara.conte@polimi.it

Michela Rossi, Dept. of Design, Politecnico di Milano, michela.rossi@polimi.it 
